\title{
NOX4 stimulates ANF secretion via activation of the Sirt1/Nrf2/ATF3/4 axis in hypoxic beating rat atria
}

\author{
ZHI-YU LI ${ }^{1,2^{*}}$, YING LIU ${ }^{1 *}$, YUE-YING WANG ${ }^{1}$, XIANG LI $^{1}$, ZHUO-NA HAN ${ }^{1}$, \\ LAN HONG $^{1}$, YING-SHUN LI ${ }^{1 *}$ and XUN CUI ${ }^{1,3}$ \\ ${ }^{1}$ Department of Physiology, School of Medical Sciences, Yanbian University, Yanji, Jilin 133002; \\ ${ }^{2}$ Medical Office, Affiliated Hospital of Yanbian University, Yanji, Jilin 133000; \\ ${ }^{3}$ Cellular Function Research Center, Yanbian University, Yanji, Jilin 133002, P.R. China
}

Received August 20, 2021; Accepted December 3, 2021

DOI: $10.3892 / \mathrm{mmr} .2022 .12600$

\begin{abstract}
Silent information regulator factor 2-related enzyme 1 (Sirt1) is involved in the regulation of cell senescence, gene transcription, energy balance and oxidative stress. However, the effect of Sirt1 on atrial natriuretic factor (ANF) secretion, especially under hypoxic conditions is unclear. The present study aimed to investigate the effect of Sirt1, regulated by NADPH oxidase 4 (NOX4), on ANF secretion in isolated beating rat atria during hypoxia. ANF secretion was analyzed using radioimmunoassays and protein expression levels were determined by western blotting and immunofluorescence staining. Intra-atrial pressure was recorded using a physiograph. Hypoxia significantly upregulated Sirt1 and nuclear factor erythroid-2-related factor 2 (Nrf2) protein expression levels, together with significantly increased ANF secretion. Hypoxia-induced protein expression of Sirt1 was significantly blocked by a NOX4 inhibitor, GLX351322, and Nrf2 protein expression levels were significantly abolished using the Sirt1 inhibitor, EX527. Hypoxia also significantly elevated the protein expression levels of phosphorylated-Akt and sequestosome 1 and significantly downregulated Kelch-like ECH-associated protein 1 protein expression levels. These effects were significantly blocked by EX527, preventing hypoxia-induced Nrf2 expression. An Nrf2 inhibitor, ML385, significantly abolished the hypoxia-induced upregulation of activating transcription factor (ATF)3, ATF4, T cell factor (TCF) 3 and TCF4/lymphoid enhancer factor 1 (LEF1) protein
\end{abstract}

Correspondence to: Professor Xun Cui or Dr Ying-shun Li, Department of Physiology, School of Medical Sciences, Yanbian University, 977 Gongyuan Road, Yanji, Jilin 133002, P.R. China

E-mail: cuixun@ybu.edu.cn

E-mail: liys@ybu.edu.cn

*Contributed equally

Key words: silent information regulator 1, nuclear factor erythroid-2-related factor 2, activating transcription factor, NADPH oxidase 4 , atrial natriuretic factor expression levels, and significantly attenuated hypoxia-induced ANF secretion. These results indicated that Sirt1 and Nrf2, regulated by NOX4, can potentially stimulate TCF3 and TCF4/LEF1 signaling via ATF3 and ATF4 activation, thereby potentially participating in the regulation of ANF secretion in beating rat atria during hypoxia. In conclusion, intervening with the Sirt1/Nrf2/ATF signaling pathway may be an effective strategy for resisting oxidative stress damage in the heart during hypoxia.

\section{Introduction}

Silent information regulator 1 (Sirt1) is an $\mathrm{NAD}^{+}$-dependent deacetylase that can deacetylate histones, non-histone proteins and various transcription factors $(1,2)$. Sirt1 is a member of the sirtuin family that has close evolutionary homology with silent mating-type information regulation 2 of yeast and serves a vital role in mammals. Moreover, Sirt1 is usually related to complex physiological processes, including oxidative stress, metabolism, apoptosis and aging (1-4). Sirt1 is highly expressed in the nuclei of embryonic mouse heart cells and its expression gradually decreases with further development of the organ. It is also expressed in both the cytoplasm and nuclei of mature cardiomyocytes $(5,6)$.

Sirt1 is involved in the regulation of aging, gene transcription, energy balance and oxidative stress in the cardiovascular system, possesses anti-inflammatory, antioxidant and anti-aging properties, and also helps resist the development of heart disease and the formation of atherosclerotic plaques (7). Furthermore, Sirt1 has been reported to be involved with ischemic/hypoxic conditions (8). Sirt1 protein expression levels are lower in the hearts of elderly patients with heart failure compared with those of young subjects (7). Sirt1 expression and left ventricular contractile function are also significantly reduced in the hearts of elderly mice, whereas overexpression of Sirtl leads to an improvement in cardiac function and a reduced mortality $(7,9)$. Moreover, it has been demonstrated that the increased cardiac apoptosis rates and infarction size induced by ischemia/reperfusion in animal models are related to reduced Sirt1 activity $(10,11)$. Studies have demonstrated that overexpression of Sirt1 in the mouse heart can promote upregulation of the antioxidant genes manganese superoxide 
dismutase and thioredoxin-1, thereby resisting oxidative stress induced by oxidants or ischemia/reperfusion and leading to the protection of cells. However, the high levels of Sirtl expression can create oxidative damage via induction of mitochondrial dysfunction $(12,13)$. In our previous study, it was observed that endothelin-1 (ET-1) could stimulate the expression of nicotinamide adenine dinucleotide phosphate oxidase 4 (NOX4), as well as hydrogen peroxide production, involved in the regulation of atrial natriuretic factor (ANF) secretion in isolated beating rat atria under normoxia or hypoxia (14). ANF is the first member of a family of cardiac natriuretic peptides that has been demonstrated to be involved in regulating body fluid volume and blood pressure homeostasis. It also possesses important anti-inflammatory and antioxidant properties that help protect cells (15).

The effect of NOX4 on Sirt1 expression and its role in regulating ANF secretion has not been fully elucidated, especially under hypoxic conditions. The aim of the present study was to therefore investigate these aspects during hypoxia in isolated perfused beating rat atria.

\section{Materials and methods}

Reagents. The NOX4 inhibitor GLX351322, Sirt1-specific inhibitor selisistat (EX527), nuclear factor erythroid-2-related factor 2 (Nrf2)-specific inhibitor ML385 and ET-1 were purchased from MedChemExpress. The dosages of these inhibitors were determined based on the $\mathrm{IC}_{50}$ concentration (GLX351322, 5 $\mu \mathrm{M}$; EX527, $123 \mathrm{nM}$; and ML385, 1.9 $\mu \mathrm{M}$ ) and the results of the pre-tests.

Isolated perfused beating left atrium preparation. A total of 90 male Sprague-Dawley rats were obtained from Laboratory Animal Center of Yanbian University (Yanji, China; weight, 260-300 g; age, 18 weeks). Rats were housed in $45-65 \%$ humidity, at a constant temperature $24 \pm 2^{\circ} \mathrm{C}$, under $12 \mathrm{~h}$ light/dark cycles and were given a free access to food and water. The rats were divided into control, hypoxia (Hy), inhibitors (GLX351322, EX527, ML385) + Hy, ET-1, EX527 + ET-1 and inhibitor only groups (36 rats were in the control group and the other groups had 6 rats/group). The animal procedures used in the present study were approved by the Animal Care and Use Committee of Yanbian University [approval no. SCXK (Ji) 2011-006] and were in accordance with the Guide for the Care and Use of Laboratory Animals published by the National Institutes of Health (16). Rats were anaesthetized by intraperitoneal injection of sodium pentobarbital (>90 mg/kg) leading to euthanasia and isolated perfused beating left atria were prepared using a previously described method (17). Briefly, the heart was rapidly removed and placed in physiological saline at $36^{\circ} \mathrm{C}$ for washing and the left atrium was dissected. An atrial cannula (length, $8 \mathrm{~cm}$; outer diameter, $1.5 \mathrm{~mm}$ ) containing two small catheters was inserted into the left atrium and the cannula was secured by ligatures. The outer tip of the atrial cannula was open to allow outflow from the atrium. The cannulated atrium was transferred to an organ chamber containing HEPES buffer. The atrium was maintained at $36^{\circ} \mathrm{C}$ and perfused with HEPES buffer solution $(1.0 \mathrm{ml} / \mathrm{min})$. The composition of the buffer was the following: $118 \mathrm{mM} \mathrm{NaCl}, 4.7 \mathrm{mM} \mathrm{KCl}, 2.5 \mathrm{mM} \mathrm{CaCl}_{2}$,
$1.2 \mathrm{mM} \mathrm{MgCl} 2,25 \mathrm{mM} \mathrm{NaHCO}, 10 \mathrm{mM}$ glucose, $10 \mathrm{mM}$ HEPES (pH 7.4 with $\mathrm{NaOH}$ ) and $0.1 \%$ bovine serum albumin (Beijing Yuanheng Shengma Institute of Biotechnology). The atria from each perfusion was stimulated at $1.5 \mathrm{~Hz}(0.3 \mathrm{msec}$; 30-40 V) with a luminal electrode.

Experimental design. The atria were perfused for $60 \mathrm{~min}$ to stabilize atrial dynamics and ANF secretion, after which formal experiments were conducted. The perfusates were collected at 2 min intervals at $4^{\circ} \mathrm{C}$ to measure ANF levels. The normoxic perfused atrium was supplied with sufficient $100 \%$ of $\mathrm{O}_{2}$ and normal HEPES buffer. The hypoxic atrial model was prepared with $100 \%$ of $\mathrm{N}_{2}$ instead of $\mathrm{O}_{2}$ and normal HEPES buffer was replaced with $\mathrm{N}_{2}$-saturated HEPES buffer. Two cycles of control (an experimental cycle $=12 \mathrm{~min}$ ) were followed by infusion of $\mathrm{N}_{2}$-saturated HEPES buffer for four cycles to determine changes in atrial dynamics and ANF levels in the perfusates. Atrial tissue was immediately frozen and stored at $-80^{\circ} \mathrm{C}$ for western blotting.

To identify the effect of NOX4 on Sirt1 and Nrf2 expression levels, as well as its role in the regulation of ANF secretion during hypoxia, another series of experiments were performed. After the control period, one cycle of treatment was followed by four cycles of treatment agent plus hypoxia infusion. The treatment agents used were: GLX351322, EX527, ML385 and ET-1 (3 nM).

Furthermore, to clarify the effective dosages of inhibitors used in the present study, 18 male Sprague-Dawley rats were selected for preliminary tests. After two control cycles, whereby the atrium was perfused with normal HEPES buffer without further treatment, one cycle of treatment was followed by four cycles of inhibitors plus hypoxia infusion.

ANF and atrial pulse pressure determination. As described previously (14), immunoreactive ANF levels in perfusates were quantified using an Iodine $\left({ }^{125} \mathrm{I}\right)$ ANF Radioimmunoassay Kit (Beijing North Institute of Biotechnology Co., Ltd.), used according to the manufacturer's protocol. The levels of secreted immunoreactive ANF were expressed as $\mathrm{ng} / \mathrm{min} / \mathrm{g}$ wet atrial tissue and the following formula was used: Changes in ANF secretion (fold)=(value of ANF-mean value of basal ANF)/mean value of basal ANF.

Intra-atrial pressure was recorded using a physiograph (RM6240EC; Chengdu Capital Instrument Factory) via a pressure transducer (YPJ01; Chengdu Capital Instrument Factory) and pulse pressure was assessed using the difference between systolic and diastolic pressures: Changes in atrial pulse pressure $($ fold $)=($ value of pulse pressure-mean value of basal pulse pressure)/mean value of basal pulse pressure.

Western blotting. As described in our previous study (14), the radioimmunoprecipitation assay (RIPA) buffer containing PMSF (cat. no. R0020; Beijing Solarbio Science \& Technology Co., Ltd.) was used to extract total protein from the left atrium. Protein concentrations were determined using the Enhanced BCA Protein Assay Kit (cat. no. P0010; Beyotime Institute of Biotechnology). After quantification, protein samples $(40 \mu \mathrm{M}$ protein/lane) were separated via SDS-PAGE on 8 or $10 \%$ gels, which was subsequently transferred to a PVDF membrane. The PVDF membranes were blocked in 5\% nonfat dry 
milk at room temperature for $2 \mathrm{~h}$, were washed three times (15 min/time) using PBS containing 0.1\% Tween-20 and were incubated with primary antibodies overnight at $4^{\circ} \mathrm{C}$. Following the primary incubation, the membranes were incubated with secondary antibody for $2 \mathrm{~h}$ at room temperature after being washed three times (15 $\mathrm{min} /$ time). Target bands were visualized using the ECL Western Blot Substrate (cat. no. 180-501; Tanon Science and Technology Co., Ltd.) using a bioanalytical imaging system. Results were semi-quantified using ImageJ version 1.48 software (National Institutes of Health). $\beta$-actin was used as the internal reference gene.

The primary antibodies used in these experiments were as follows: Sirt1 (1:1,000; cat. no. DF6033; Affinity Biosciences), Nrf2 (1:1,000; cat. no. bs-1074R; BIOSS), total-Akt (1:10,000; cat. no. ab179463; Abcam), phosphorylated (p)-Akt (1:1,000; cat. no. AF0016; Affinity Biosciences), sequestosome 1 (p62; 1:1,000; cat. no. bs55207R; BIOSS), Kelch-like ECH-associated protein 1 (Keap1; 1:5,000; cat. no. 10503-2-AP; Wuhan Sanying Biotechnology), T cell factor (TCF)3 (1,000; cat. no. DF4573; Affinity Biosciences) and TCF4 (1:1,000; cat. no. DF7622; Affinity Biosciences), lymphoid enhancer factor 1 (LEF1; 1:1,000; cat. no. DF7570; Affinity Biosciences), activating transcription factor (ATF)3 (1:1,000; cat. no. DF6660; Affinity Biosciences) and ATF4 (1:1,000; cat. no. DF6008; Affinity Biosciences) and $\beta$-actin (1:1,000; cat. no. BM3873; Boster Biological Technology). The secondary antibody used was HRP-conjugated goat anti-rabbit IgG (Heavy chain + Light chain), which was obtained from Nachuan Biotech Co. (1:3,000; cat. no. AP132P).

Immunofluorescence staining. The fresh atrial tissue was fixed in $4 \%$ paraformaldehyde at room temperature for $24 \mathrm{~h}$. The deparaffinized and rehydrated (using a descending ethanol gradient and distilled water; $5 \mathrm{~min} / \mathrm{step})$ atrial tissue sections $(5.0-\mu \mathrm{m}$ sections) were immersed in $0.1 \mathrm{~mol} / \mathrm{l}$ sodium citrate solution for antigen retrieval and placed in a microwave oven to heating by medium-high fire $\left(80-90^{\circ} \mathrm{C}\right)$ for $8 \mathrm{~min}$ until the solution had been boiled and the samples were left in the oven for $10 \mathrm{~min}$ after the fire had been switched off. After repeating this step three times the solution was cooled naturally. The sections were immersed in PBS to washing three times (5 min/time) and were then incubated with $0.1 \%$ Triton $\mathrm{X}-100$ at room temperature for $15 \mathrm{~min}$ (cat. no. ZLI-9308; OriGene Technologies, Inc.). The penetrated sections were washed in PBS three times ( $5 \mathrm{~min} / \mathrm{time}$ ) using a decoloring shaker and then blocked by using $10 \%$ goat-derived antibody blocking solution (cat. no. G2010; Wuhan Servicebio Technology Co., Ltd.) at room temperature for $30 \mathrm{~min}$. After removal of the blocking solution, three antibodies against Sirt1 (1:200; cat. no. DF6033; Affinity Biosciences), Nrf2 (1:200; cat. no. bs-1074R; BIOSS) and LEF1 (1:200; cat. no. DF7570; Affinity Biosciences) were added to the sections and incubated overnight at $4^{\circ} \mathrm{C}$. Subsequently, fluorescent dye-labelled secondary antibodies [Cy3 conjugated with goat anti-rabbit IgG (1:200; cat. no. GB21303; Wuhan Servicebio Technology Co., Ltd.) and Alexa Fluor ${ }^{\circledR}$ 488-conjugated with goat anti-rabbit IgG (1:200; cat. no. GB25303; Wuhan Servicebio Technology Co., Ltd.)] were added to the sections, which were incubated at room temperature for $30 \mathrm{~min}$ in the dark. Subsequently, samples were incubated for a further $8 \mathrm{~min}$ with $2 \mu \mathrm{g} / \mathrm{ml}$ DAPI (cat. no. G1012; Wuhan Servicebio Technology Co., Ltd.) at room temperature for cell nuclear staining. Finally, Fluoromount-G (cat. no. G1401; Wuhan Servicebio Technology Co., Ltd.) was added and covered with a cover slide, according to the manufacturer's instructions. The sections were mounted in an antifade mounting medium. An inverted fluorescent biological microscope was used to image the samples (BDS 400; ChongQing Optec Instrument Co., Ltd.).

Statistical analysis. Prism software (version 7; GraphPad Software, Inc.) was used to analyze the data. All data are presented as the mean $\pm \mathrm{SE}$ and experiments were repeated six times. Significant differences were statistically compared using one-way ANOVA, followed by Bonferroni's multiple comparisons test for more than two groups. $\mathrm{P}<0.05$ was considered to indicate a statistically significant difference.

\section{Results}

Effects of NOX4 inhibition on Sirtl and Nrf2 expression levels during hypoxia. To clarify the effective dosages of inhibitors used in the present study, two dosages of inhibitors were used in the preliminary tests. The results demonstrated that the optimal doses of GLX351322, EX3527 and ML385 were 35, 0.25 and $10 \mu \mathrm{M}$, respectively, rather than their $\mathrm{IC}_{50}$ concentrations (GLX351322, $5 \mu \mathrm{M}$; EX527, $123 \mathrm{nM}$; and ML385, $1.9 \mu \mathrm{M}$ ), which significantly inhibited the hypoxia-induced increase of atrial ANF secretion (Fig. S1). Therefore, the effective dosages of these inhibitors were used in subsequent experiments. Furthermore, to clarify the effect of NOX4 on Sirt1 and Nrf2 expression and its role in the regulation of ANF secretion under hypoxic conditions, experiments were performed with a NOX4 inhibitor in isolated beating rat hypoxic atria. The results demonstrated that compared with the control group hypoxia significantly increased ANF secretion and inhibited atrial pulse pressure (Fig. 1A and B) concomitantly with the significant upregulation of Sirt1 and Nrf2 protein expression levels (Fig. 1C-F). The hypoxia-induced protein expression of Sirt1 was significantly inhibited by treatment with the NOX4 inhibitor, GLX351322, compared with the hypoxia group, whereas Nrf2 protein expression induced by hypoxia was significantly abolished by EX527 treatment, an inhibitor of Sirt1, compared with the hypoxia group. These results indicated that NOX4 may stimulate Sirt1 activation and its downstream factor Nrf2 in beating rat atria under hypoxic conditions.

Effect of Sirtl inhibition on p-Akt, p62 and Keapl expression under hypoxia. To explore the mechanism by which Sirt1 regulates Nrf2 protein expression levels, the effect of Sirt1 inhibition on the protein expression levels of p-Akt, p62 and Keap1 induced by ET-1 and hypoxia were examined. The results demonstrated that the protein expression levels of p-Akt and p62 were significantly upregulated by exogenous ET-1 treatment, whereas Keap1 protein expression levels were significantly downregulated in the ET-1 group compared with the control (Fig. 2). The ET-1-mediated effects on p-Akt, p62 and Keap1 were significantly eliminated with EX527 treatment compared with the control. Moreover, the hypoxia-induced significant upregulation of p-Akt and p62 protein expression levels, as well as the significant downregulation of Keap1 protein expression levels compared with the control, were also significantly 
A

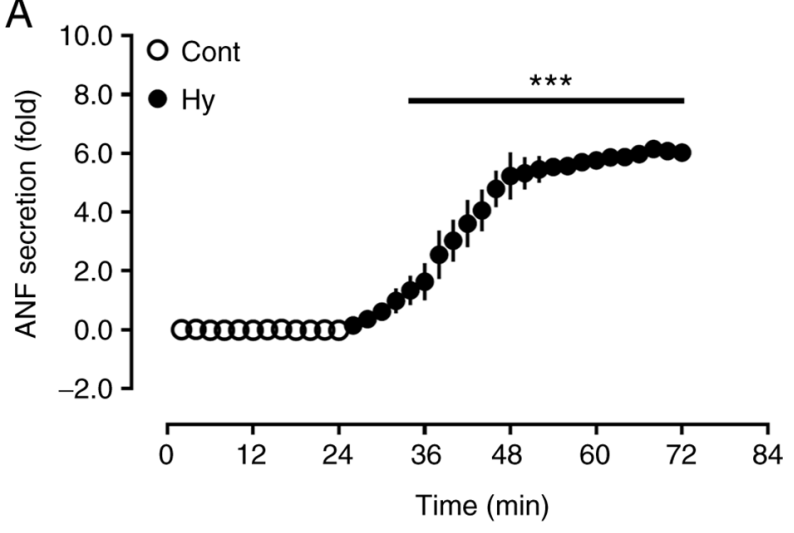

C
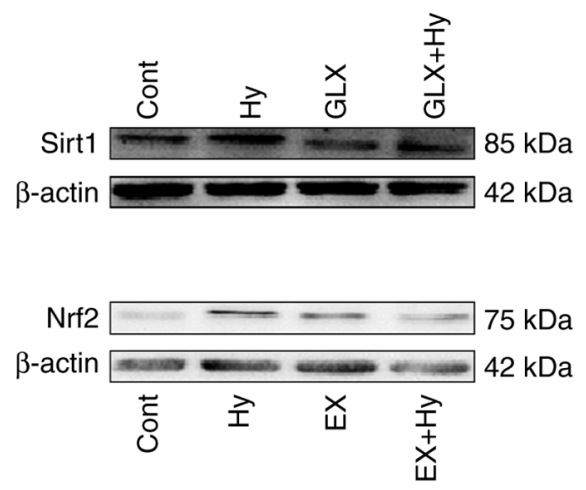

$E$

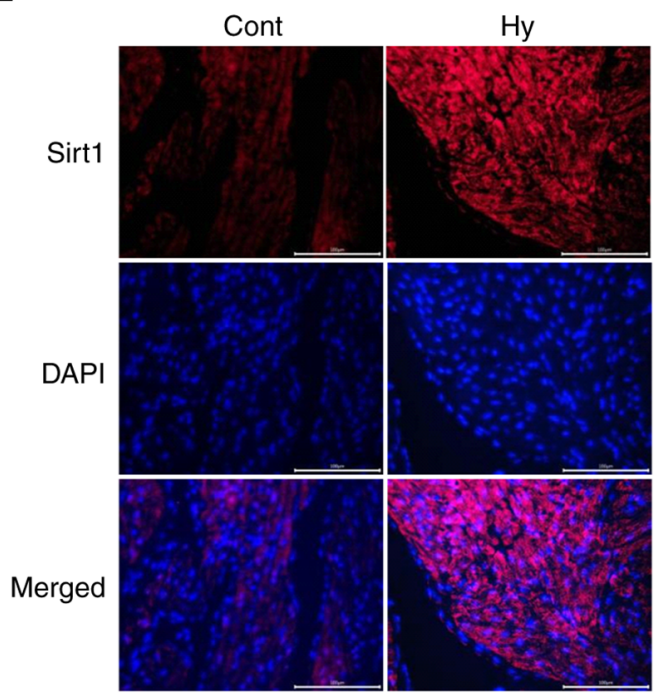

B
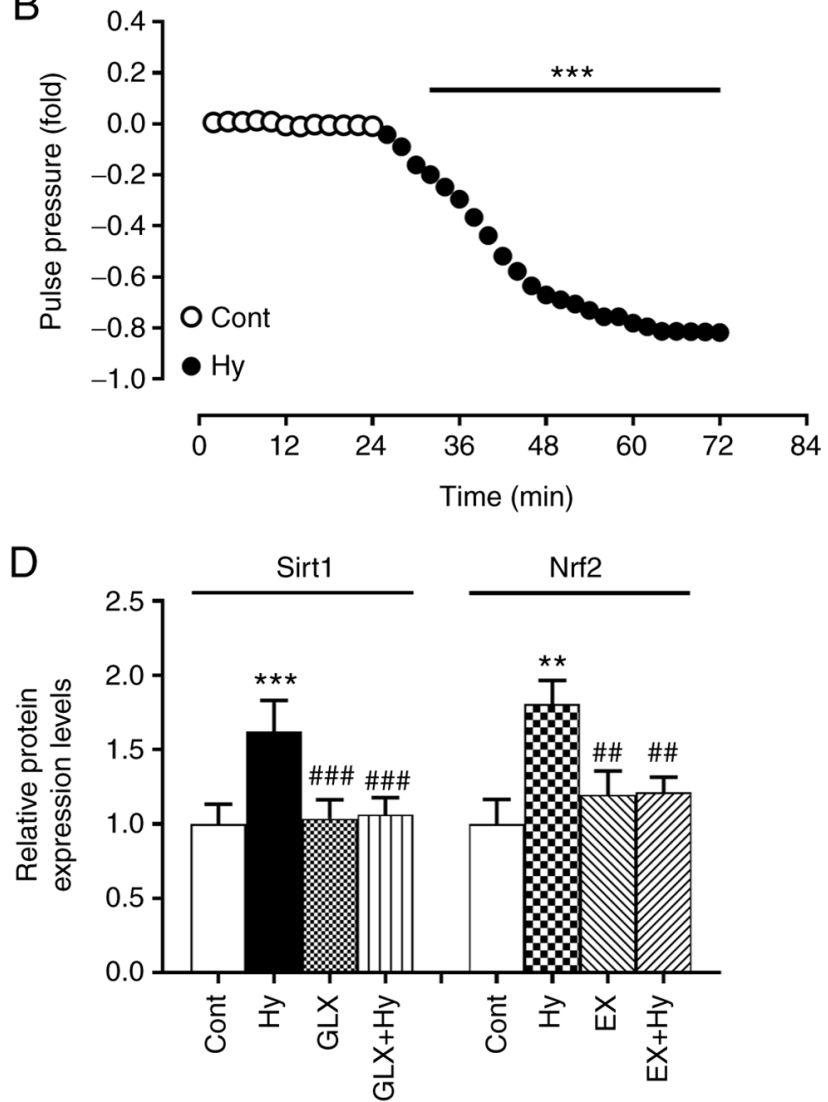

$\mathrm{F}$

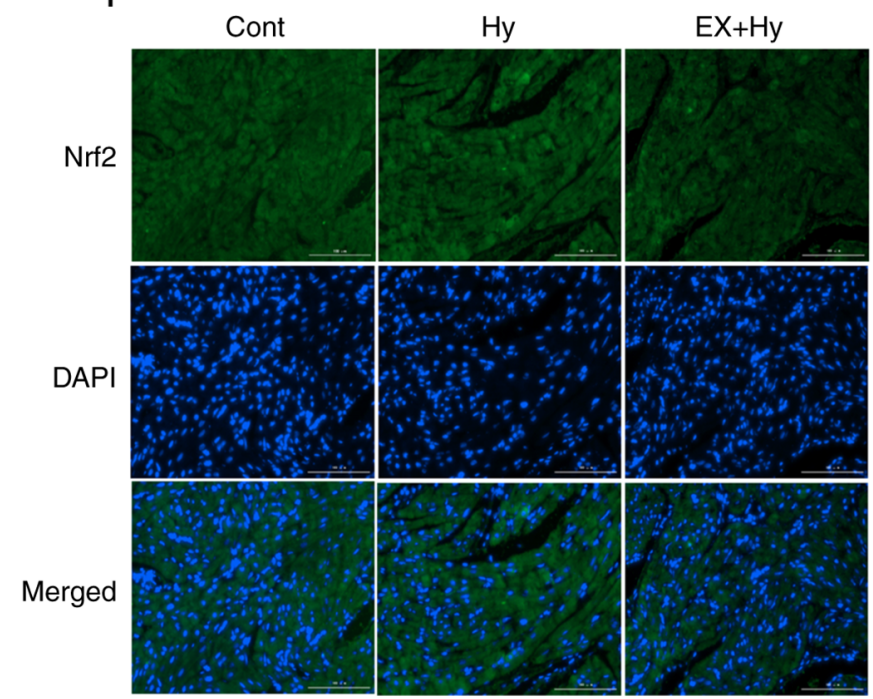

Figure 1. Effects of hypoxia on ANF secretion, pulse pressure and the protein expression levels of Sirt1 and Nrf2 in beating rat atria. (A) ANF secretion; (B) pulse pressure; (C-F) protein expression levels of Sirt1 and Nrf2. Protein expression levels have been analyzed using western blotting, quantification of protein levels and immunofluorescence staining (scale bar, $100 \mu \mathrm{m}$ ). Data are presented as the mean $\pm \mathrm{SE}$. A and $\mathrm{B}, \mathrm{n}=6 ; \mathrm{B}$ and $\mathrm{C}, \mathrm{n}=5$. ${ }^{* *} \mathrm{P}<0.01{ }^{* * * *} \mathrm{P}<0.001 \mathrm{vs}$. control; ${ }^{\# \#} \mathrm{P}<0.01,{ }^{\# \# \#} \mathrm{P}<0.001$ vs. Hy. ANF, atrial natriuretic factor; sirt1, silent information regulator 1; Nrf2, nuclear factor erythroid-2-related factor 2; cont, control; hy, hypoxia; GLX, GLX351322; EX, EX527.

reversed by EX527 treatment in the EX + Hy group compared with the hypoxia group. These results suggested that Sirt1 may regulate Nrf2 protein expression levels via the downregulation of Keap1 and the activation of Akt and p62.

Effect of Sirtl and Nrf2 inhibition on ANF secretion during hypoxia. To determine the effect of Sirt1 and Nrf2 on ANF secretion in beating hypoxic atria, inhibitors of Sirt1 and Nrf2 were used. Hypoxia significantly stimulated the atrial secretion of ANF (Fig. 3A and C) and also significantly suppressed pulse pressure, compared with the control group (Fig. 3B and D). The hypoxia-induced increase in ANF secretion was significantly attenuated by the Sirt1 and Nrf2 inhibitors, EX527 and ML385, respectively, compared with the hypoxia group. The atrial pulse pressure induced by hypoxia was not significantly affected by EX527 and ML385. These data suggested that 
A
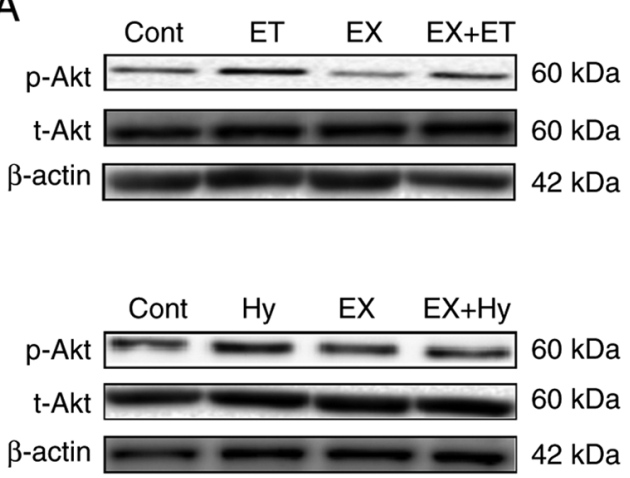

C

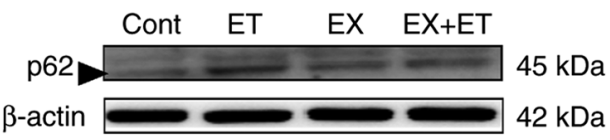

E
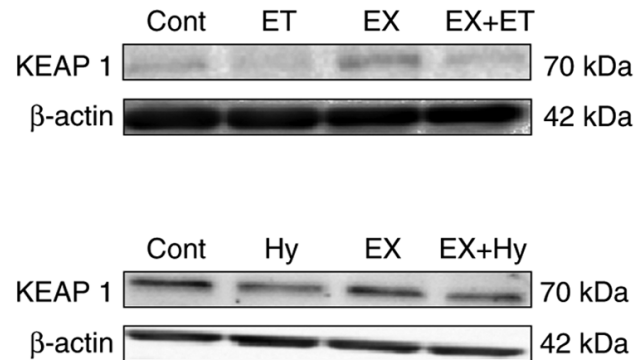

B

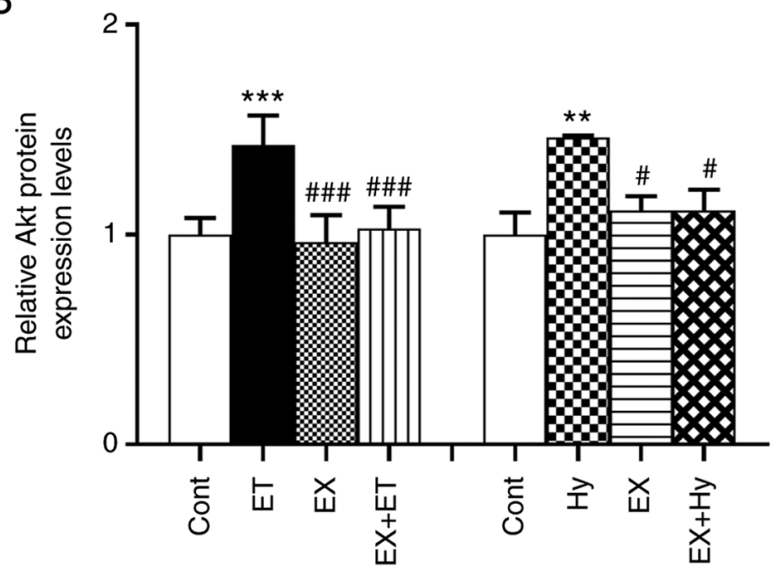

D
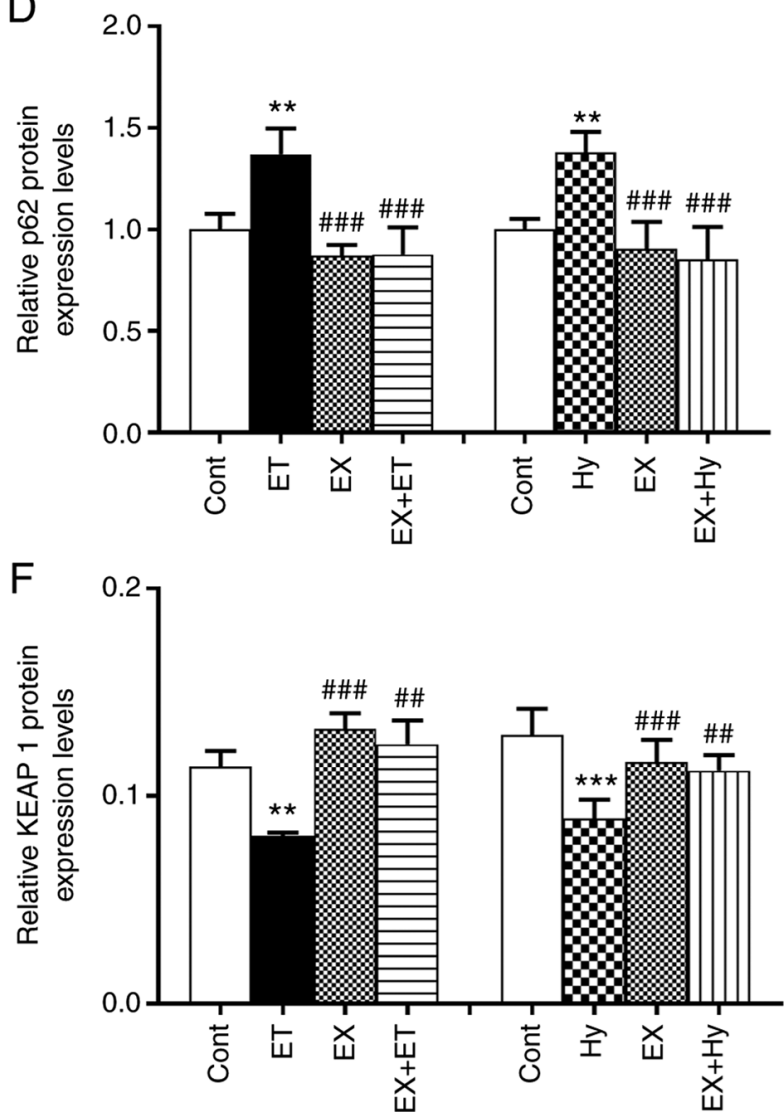

Figure 2. Effects of Sirt1 inhibitor on the levels of p-Akt, p62 and Keap1 induced by ET-1 (under normoxia) or hypoxia in beating rat atria (A, C and E) Representative western blots (in C, the band next to the p62 band is a miscellaneous band; size, $\sim 47 \mathrm{kDa}$ ). (B, D and F) Semi-quantificat ion of protein expression levels. Data are presented as the mean $\pm \mathrm{SE} . \mathrm{n}=5 .{ }^{* * *} \mathrm{P}<0.01,{ }^{* * * *} \mathrm{P}<0.001$ vs. control; ${ }^{\#} \mathrm{P}<0.05 \mathrm{~m}{ }^{\# \#} \mathrm{P}<0.01,{ }^{\# \# *} \mathrm{P}<0.001 \mathrm{vs}$. hypoxia. p62, sequestosome 1; Keap1, Kelch-like ECH-associated protein 1; cont, control; ET/ET-1, endothelin-1; hy, hypoxia; EX, EX527; t, total; p, phosphorylated.

Sirt1 and Nrf2, controlled by NOX4, may be involved in the regulation of ANF secretion during hypoxia.

Effect of hypoxia on the regulation of ATF3 and ATF4 protein expression levels. Due to the role of Nrf2 in the regulation of ATF activity (18), the present study further investigated the effects of hypoxia on ATF3 and ATF4 expression levels. The results demonstrated that hypoxia significantly upregulated both ATF3 and ATF4 protein expression levels, compared with the control (Fig. 4). In the presence of the Nrf2 inhibitor, ML385, the hypoxia-induced upregulation of ATF3 and ATF4 protein expression was not observed. The basal levels of ATF3 and ATF4 protein expression levels were not affected by ML385 alone. These data indicated that the activities of ATF3 and ATF4 may be controlled by Nrf2 during hypoxia.

Effect of hypoxia on TCF3, TCF4 and LEF1 protein expression levels. A previous study reported the influence of ATF on TCF/LEF1, as well as the role of LEF1 in regulating ANF promoter activity (19). Therefore, the effects of hypoxia on TCF3, TCF4 and LEF1 protein expression levels were examined. The results demonstrated that hypoxia significantly elevated TCF3, TCF4 and LEF1 protein expression levels compared with the control (Fig. 5).ML385 significantly reduced 

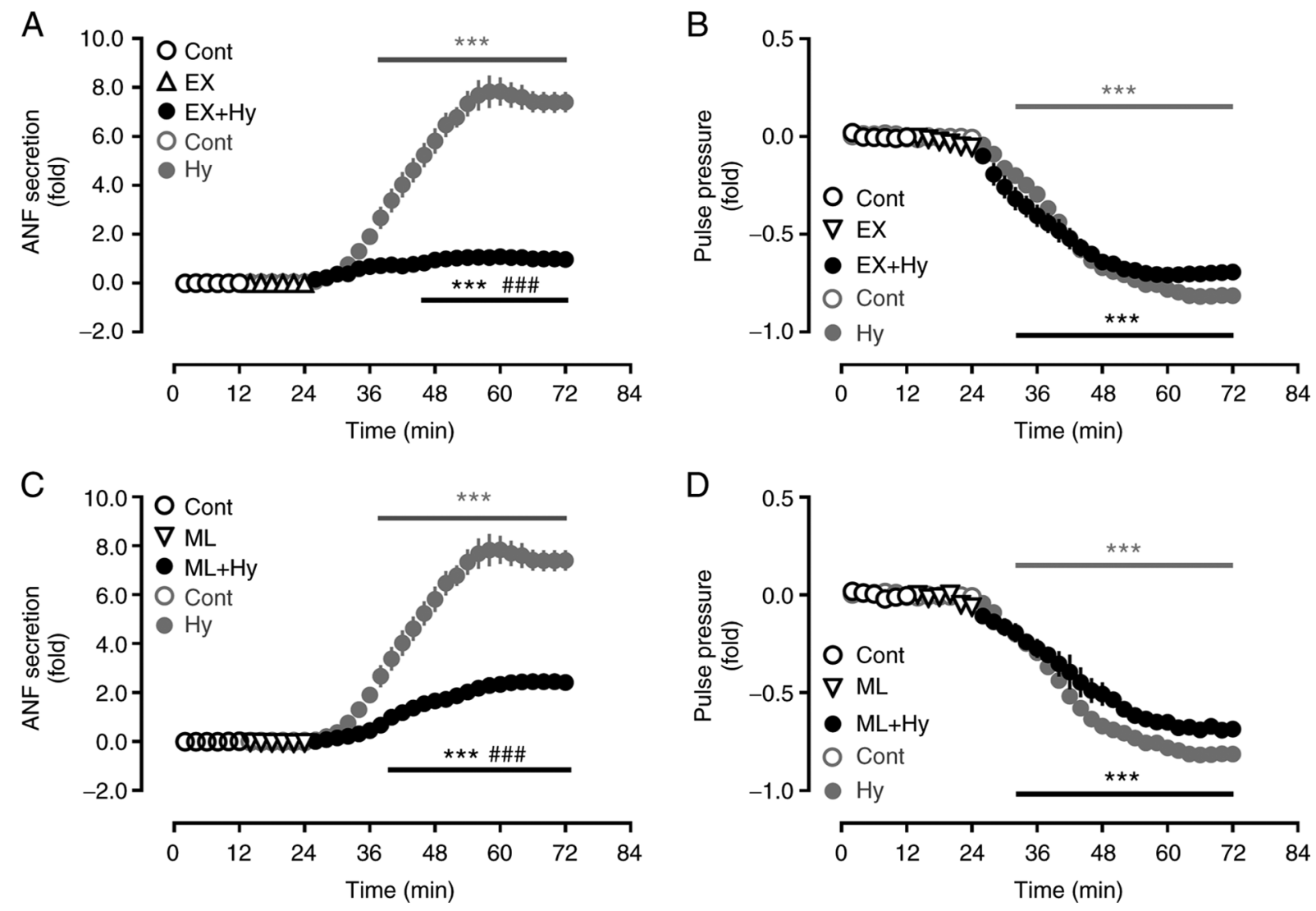

Figure 3. Effects of silent information regulator 1 and nuclear factor erythroid-2-related factor 2 inhibitors on (A and C) ANF secretion and (B and D) pulse pressure in beating rat atria under hypoxia. Data are presented as the mean $\pm \mathrm{SE}$. $\mathrm{n}=6 .{ }^{* * * *} \mathrm{P}<0.001$ vs. control; ${ }^{\# \# \#} \mathrm{P}<0.001$ vs. hypoxia. ANF, atrial natriuretic factor; cont, control; hy, hypoxia; EX, EX527; ML, ML385.
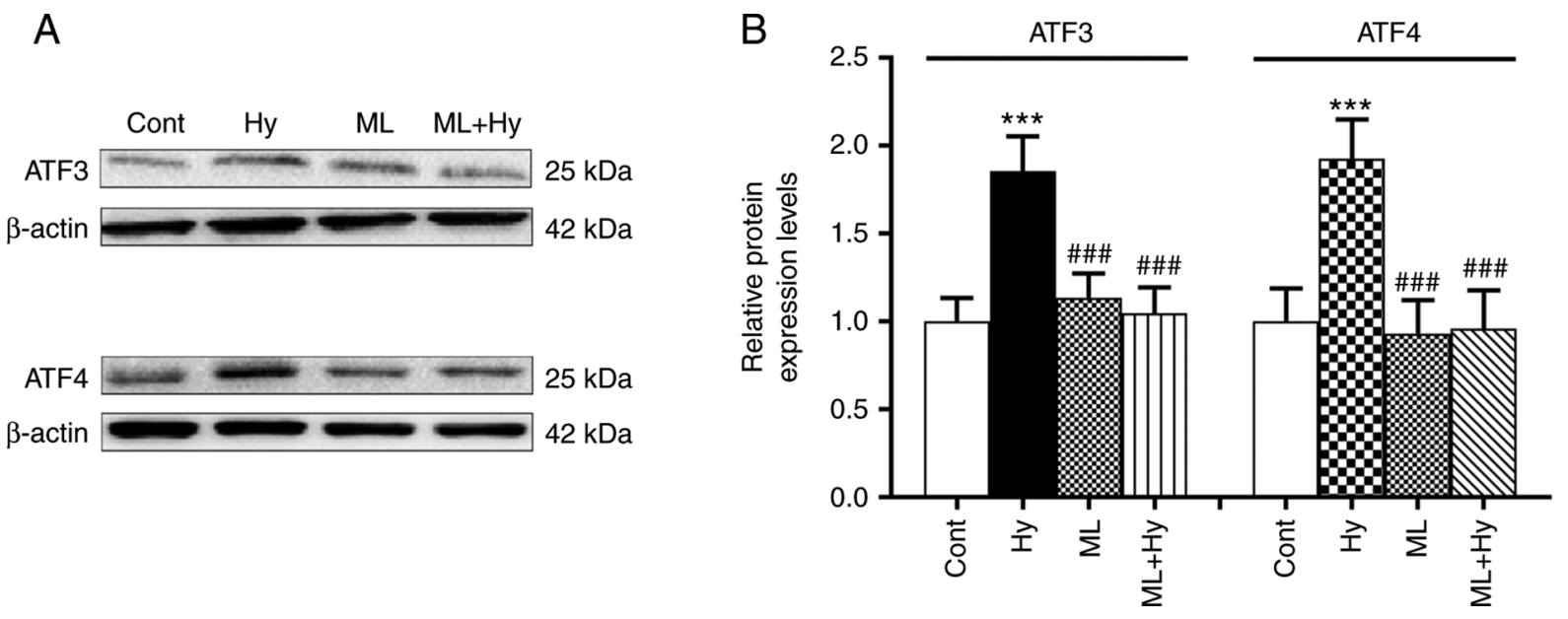

Figure 4. Effects of nuclear factor erythroid-2-related factor 2 inhibitor on ATF3 and ATF4 protein expression induced by hypoxia in beating rat atria. (A) Representative western blots. (B) Quantification of protein expression levels. Data are presented as the mean $\pm \mathrm{SE}$. $\mathrm{n}=5$. ${ }^{* * *} \mathrm{P}<0.001 \mathrm{vs}$. control; ${ }^{\# \#} \mathrm{P}<0.001$ vs. hypoxia. ATF, activating transcription factor; cont, control; hy, hypoxia; ML, ML385.

the effect of hypoxia on these three proteins, compared with the hypoxia group. The results also demonstrated that ML385 also significantly attenuated the hypoxia-induced increase in ANF secretion, compared with the hypoxia group (Fig. 6A). The hypoxia-induced inhibition of atrial pulse pressure was not significantly changed by ML385 treatment (Fig. 6B). These results indicated that $\mathrm{Nrf} 2$ may be involved in regulating ANF secretion via the activation of TCF/LEF1 signaling.

\section{Discussion}

In the present study, hypoxic conditions resulted in the significant upregulation of Sirt1 and Nrf2 protein expression levels, along with significantly increased ANF secretion, in isolated beating rat atria. The hypoxia-induced expression of Sirt1 was blocked by an inhibitor of NOX4 and Nrf2 expression was abolished by the Sirt1 inhibitor. Hypoxia also significantly 
A

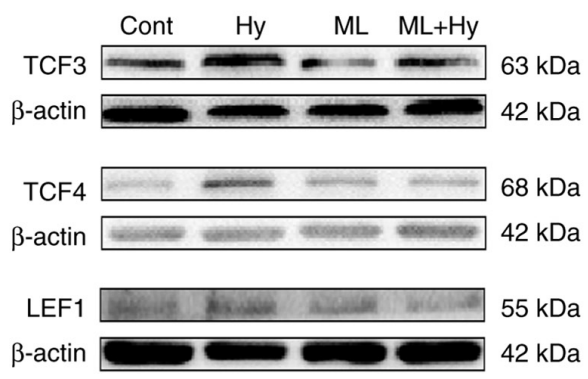

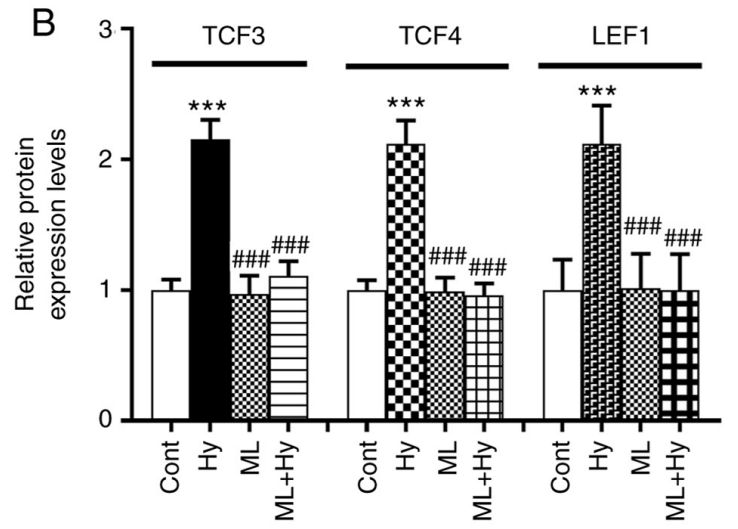

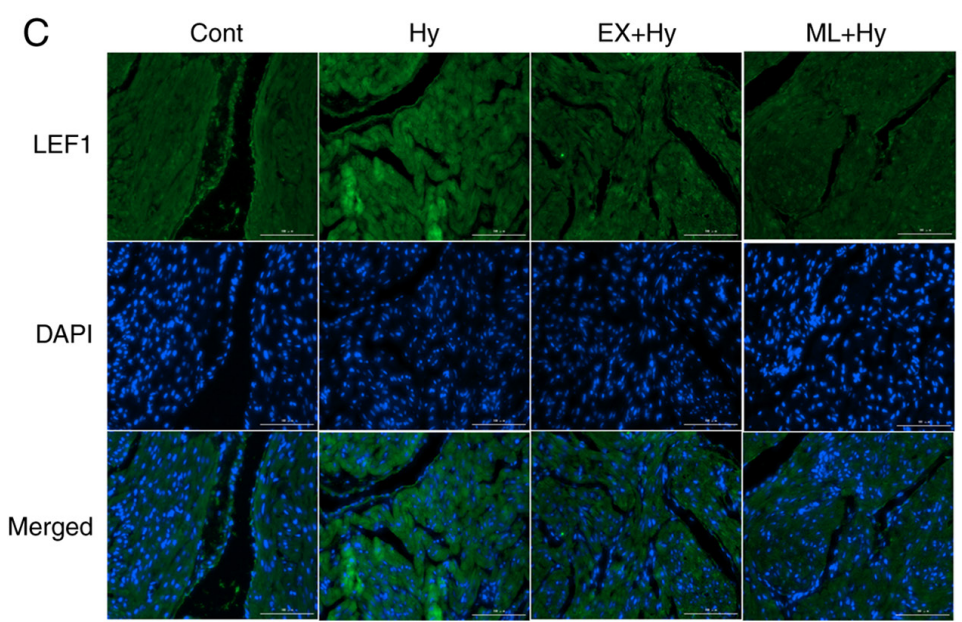

Figure 5. Effect of nuclear factor erythroid-2-related factor 2 inhibitor on TCF3, TCF4 and LEF1 protein expression induced by hypoxia in beating rat atria. (A) Representative western blots. (B) Quantification of protein expression levels. (C) LEF1 protein expression was detected using immunofluorescence staining (scale bar, $100 \mu \mathrm{m}$ ). Data are presented as the mean $\pm \mathrm{SE} . \mathrm{n}=5 .{ }^{* * *} \mathrm{P}<0.001$ vs. control; ${ }^{\# \#} \mathrm{P}<0.001$ vs. hypoxia. TCF, T-cell factor; LEF1, lymphoid enhancer factor 1; cont, control; hy, hypoxia; ML, ML385.
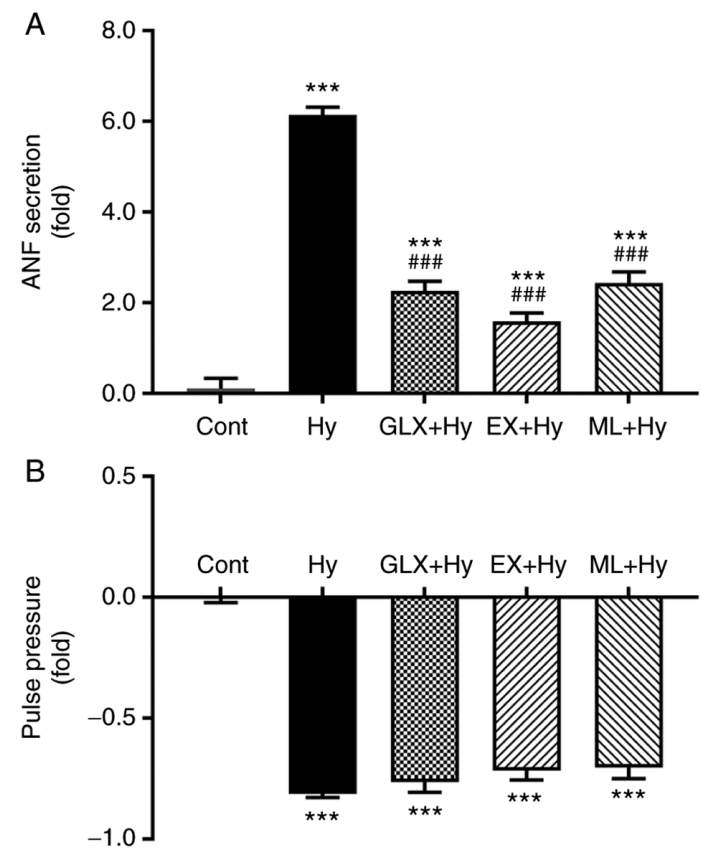

Figure 6. Effects of NADPH oxidase 4, silent information regulator 1 and nuclear factor erythroid-2-related factor 2 inhibitors on ANF secretion and pulse pressure in beating rat atria during hypoxia. (A) ANF secretion. (B) Pulse pressure. Data are presented as the mean \pm SE. $n=5 .{ }^{* * * *} \mathrm{P}<0.001$ vs. control; ${ }^{\# \# \#} \mathrm{P}<0.001$ vs. hypoxia. ANF, atrial natriuretic factor; cont, control; hy, hypoxia; GLX, GLX351322; EX, EX527; ML, ML385. elevated the protein expression levels of p-AKT and p62, whereas Keap1 protein expression levels were significantly downregulated. Moreover, these effects were blocked by treatment with a Sirt1 inhibitor, which prevented hypoxia-induced Nrf2 protein expression. Furthermore, the Nrf2 inhibitor abolished the hypoxia-induced upregulation of ATF3, ATF4 and TCF3, as well as TCF4/LEF1 protein expression levels, accompanied by the significant attenuation of hypoxia-induced ANF secretion. These results suggested that NOX4 possibly regulated Sirt1 and its downstream protein Nrf2, which stimulated TCF3 and TCF4/LEF1 signaling by activating ATF3 and ATF4, thereby participating in the regulation of ANF secretion during hypoxia (Fig. 7).

Sirtl has been reported to be localized to both the cytoplasm and nucleus under basal conditions and is shuttled to the nucleus in response to certain stressors in the heart (5). Acute ischemic preconditioning, pressure overload, nutrient starvation and exercise, can also upregulate Sirtl expression in the heart $(12,13)$. Furthermore, Sirt1 prevents apoptosis of cardiomyocytes and protects the heart from ischemia/reperfusion-induced damage $(20,21)$. Sirt1 acts as an upstream signaling molecule for the transcription factor $\mathrm{Nrf} 2$, which serves a role in resisting ischemia/reperfusion injury via activation of Nrf2 and its downstream signaling pathway $(22,23)$. The results of the present study demonstrated that hypoxia can significantly increase ANF secretion and significantly inhibit atrial pulse pressure, as well 


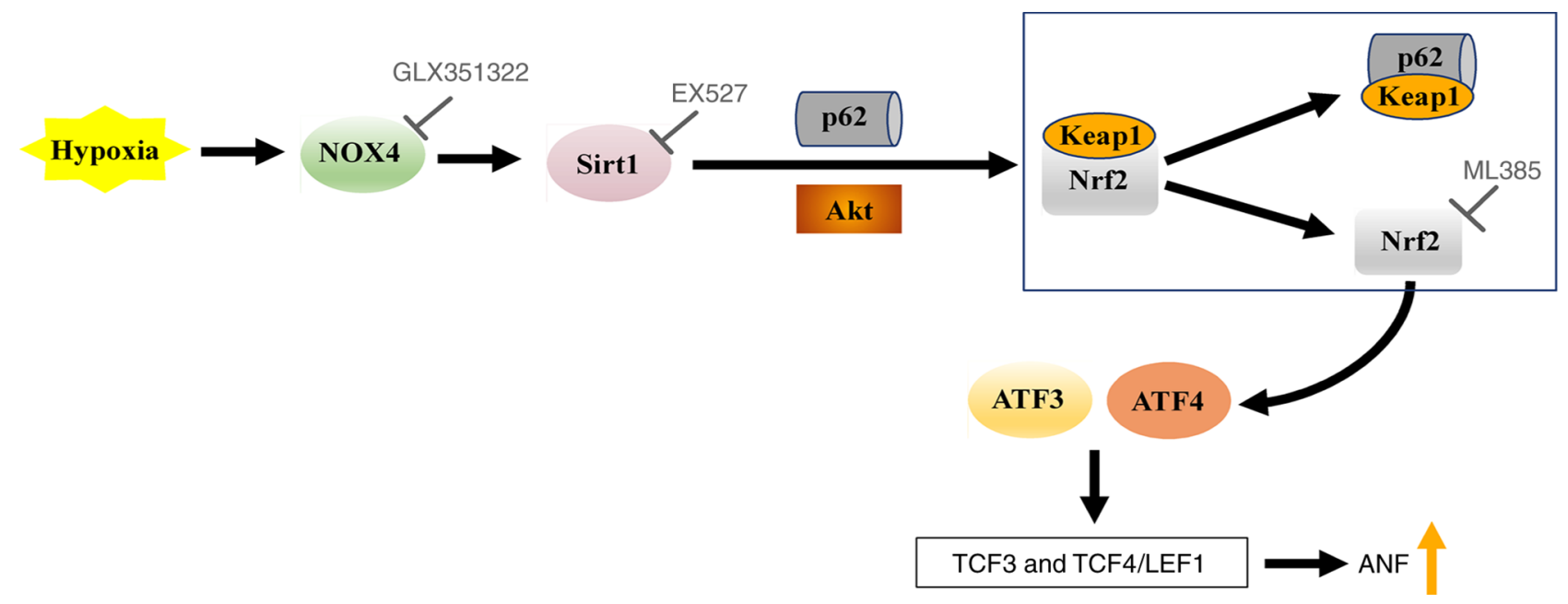

Figure 7. Schematic of the NOX4/Sirt1/Nrf2 signaling pathway, which regulates ANF secretion during hypoxia. NOX4, NADPH oxidase 4; Sirt1, silent information regulator 1; Nrf2, nuclear factor erythroid-2-related factor 2; p62, sequestosome 1; Keap1, Kelch-like ECH-associated protein 1; ATF, activating transcription factor; TCF, T-cell factor; LEF1, lymphoid enhancer factor 1; ANF, atrial natriuretic factor.

as significantly upregulate Sirt1 and Nrf2 protein expression levels. This upregulation was significantly blocked by treatment with a NOX4 inhibitor. Adding a Sirt1 antagonist also abolished Nrf2 protein expression levels during hypoxic conditions without significant changes to atrial pulse pressure. Moreover, the significantly upregulated levels of p-Akt and p62, as well as the significantly downregulated levels of Keap1, induced by exogenous ET-1 under normoxic or hypoxic conditions were also significantly abolished using the Sirtl antagonist. These results indicated that Sirt1 possibly downregulated Keap1 by activating Akt and p62, thereby upregulating Nrf2 protein expression. The data in the present study are consistent with the aforementioned cited studies and support the notion that p62 aggregation leads to the recruitment of Keap1 for the degradation and release of Nrf2 into the nucleus (24).

ATF3 and ATF4, members of the transcription factor ATF/cyclic AMP-reactive element binding protein family, are expressed in the cardiovascular system in response to a variety of stimuli, including pathological stressors, such as pressure overload, stretch, ischemia/reperfusion, ischemic preconditioning, hypoxia, ET-1 and $\mathrm{H}_{2} \mathrm{O}_{2}(25,26)$. Previously, it has been confirmed that $\mathrm{Nrf} 2$ can resist oxidative stress and protects the heart via activation of ATF (18). Moreover, members of the ATF family participate in the regulation of the transcriptional processes via activation of the $\mathrm{Wnt} / \beta$-catenin signaling pathway downstream effectors TCF and LEF (27). The roles of TCF and LEF in the regulation of ANF promoter activity and transcription were also demonstrated in rat cultured cardiomyocytes (19). In the present study, the results demonstrated that hypoxia not only significantly upregulated the protein expression levels of ATF3 and ATF4, but also significantly increased the protein expression levels of TCF3, TCF4 and LEF1, which may lead to the promotion of ANF secretion. The hypoxia-induced increases in ATF3, ATF4, TCF3, TCF4 and LEF1 were significantly abolished by adding a specific antagonist of $\mathrm{Nrf} 2$, accompanied by a significant attenuation of hypoxia-augmented secretion of ANF. These results demonstrated that $\mathrm{Nrf2}$, controlled by Sirt1, may stimulate TCF3 and TCF4/LEF1 via activation of ATF3 and
ATF4, leading to promoted ANF secretion in beating rat atria during hypoxia. The results of the present study support the previously aforementioned studies. In our previous study, we demonstrated that endogenous ET-1 participates in the regulation of ANF secretion by regulating the NOX4/proto-oncogene tyrosine-protein kinase Src signaling pathway in rat beating atria during hypoxia (14). Therefore, it can be hypothesized that the regulation of hypoxia-induced ANF secretion by NOX4/Sirt1/Nrf2 in the present study is another important regulatory mechanism by which endogenous ET-1 regulates hypoxia-induced ANF secretion via NOX4.

In summary, it was demonstrated that hypoxia significantly upregulated the expression levels of Sirtl and its downstream molecule Nrf2 via NOX4, which may have activated TCF3 and TCF4/LEF1 via ATF3 and ATF4 signaling. This cascade ultimately resulted in increased ANF secretion. Therefore, the present study indicated that the Sirt1/Nrf2/ATF axis is potentially involved in a cardioprotective mechanism against oxidative stress damage during hypoxia. A limitation of the present study is the lack of inhibitors for ATF and TCF/LEF1, it is therefore necessary to further verify the results of the study by knockout or knockdown of these factors in future work. Intervening with the Sirt1/Nrf2/ATF signaling pathway may be useful for the prevention of hypoxic heart disease.

\section{Acknowledgements}

Not applicable.

\section{Funding}

The present study was supported by the National Natural Science Foundation of China (grant nos. 81960099 and 81660089).

\section{Availability of data and materials}

The datasets used and/or analyzed during the current study are available from the corresponding authors on reasonable request. 


\section{Authors' contributions}

ZYL and YL performed the atrial perfusion experiments and ANP measurements. YYW and XL performed the western blotting analysis. ZNH and $\mathrm{LH}$ performed the statistical analysis of the experimental data. XC and YSL designed the experiments and wrote the manuscript. XC and YSL confirm the authenticity of all the raw data. All authors read and agreed to the final manuscript.

\section{Ethics approval and consent to participate}

The animal procedures used in the present study were approved by the Animal Care and Use Committee of Yanbian University [approval no. SCXK (Ji) 2011-006] and were in accordance with the Guide for the Care and Use of Laboratory Animals published by the National Institutes of Health (16).

\section{Patient consent for publication}

Not applicable.

\section{Competing interests}

The authors declare that they have no competing interests.

\section{References}

1. Kumari S, Chaurasia SN, Nayak MK, Mallick RL and Dash D: Sirtuin inhibition induces apoptosis-like changes in platelets and thrombocytopenia. J Biol Chem 290: 12290-12299, 2015.

2. Boutant $M$ and Canto C: SIRT1 metabolic actions: Integrating recent advances from mouse models. Mol Metab 3: 5-18, 2013.

3. Tsai KL, Cheng YY, Leu HB, Lee YY, Chen TJ, Liu DH and Kao CL: Investigating the role of Sirt1-modulated oxidative stress in relation to benign paroxysmal positional vertigo and Parkinson's disease. Neurobiol Aging 36: 2607-2616, 2015.

4. Poulose N and Raju R: Sirtuin regulation in aging and injury. Biochim Biophys Acta 1852: 2442-2455, 2015.

5. Tanno M, Sakamoto J, Miura T, Shimamoto K and Horio Y: Nucleocytoplasmic shuttling of the NAD+-dependent histone deacetylase SIRT1. J Biol Chem 282: 6823-6832, 2007.

6. Moynihan KA, Grimm AA, Plueger MM, Bernal-Mizrachi E, Ford E, Cras-Méneur C, Permutt MA and Imai SI: Increased dosage of mammalian Sir2 in pancreatic beta cells enhances glucosestimulated insulin secretion in mice. Cell Metab 2: 105-117, 2005.

7. D'Onofrio N, Servillo L and Balestrieri ML: SIRT1 and SIRT6 signaling pathways in cardiovascular disease protection. Antioxid Redox Signal 28: 711-732, 2018.

8. Meng X, Tan J, Li M, Song S, Miao Y and Zhang Q: Sirt1: Role under the condition of ischemia/hypoxia. Cell Mol Neurobiol 37: $17-28,2017$.

9. Gu XS, Wang ZB, Ye Z, Lei JP, Li L, Su DF and Zheng X: Resveratrol, an activator of SIRT1, upregulates AMPK and improves cardiac function in heart failure. Genet Mol Res 13: 323-335, 2014

10. Cattelan A, Ceolotto G, Bova S, Albiero M, Kuppusamy M, De Martin S, Semplicini A, Fadini GP, de Kreutzenberg SV and Avogaro A: NAD(+)-dependent SIRT1 deactivation has a key role on ischemia-reperfusion-induced apoptosis. Vascul Pharmacol 70: 35-44, 2015.
11. Shalwala M, Zhu SG, Das A, Salloum FN, Xi L and Kukreja RC: Sirtuin 1 (SIRT1) activation mediates sildenafil induced delayed cardioprotection against ischemia-reperfusion injury in mice. PLoS One 9: e86977, 2014

12. Alcendor RR, Gao S, Zhai P, Zablocki D, Holle E, Yu X, Tian B, Wagner T, Vatner SF and Sadoshima J: Sirt1 regulates aging and resistance to oxidative stress in the heart. Circ Res 100: 1512-1521, 2007.

13. Hsu CP, Zhai P, Yamamoto T, Maejima Y, Matsushima S, Hariharan N, Shao D, Takagi H, Oka S and Sadoshima J: Silent information regulator 1 protects the heart from ischemia/ reperfusion. Circulation 122: 2170-2182, 2010.

14. Wu CZ, Li X, Hong L, Han ZN, Liu Y, Wei CX and Cui X: NOX4/Src regulates ANP secretion through activating ERK1/2 and Akt/GATA4 signaling in beating rat hypoxic atria. Korean J Physiol Pharmacol 25: 159-166, 2021.

15. Kim HY, Cho KW, Xu DY, Kang DG and Lee HS: Endogenous ACh tonically stimulates ANP secretion in rat atria. Am J Physiol Heart Circ Physiol 305: H1050-H1056, 2013.

16. National Research Council (US) Committee for the Update of the Guide for the Care and Use of Laboratory Animals: Guide for the Care and Use of Laboratory Animals. 8th edition. National Academies Press, Washington, DC, 2011.

17. Li X, Han ZN, Liu Y, Hong L, Cui BR and Cui X: Endogenous ET-1 promotes ANP secretion through activation of COX2-L-PGDS-PPAR $\gamma$ signaling in hypoxic beating rat atria. Peptides 122: 170150, 2019.

18. Chen QM and Maltagliati AJ: Nrf2 at the heart of oxidative stress and cardiac protection. Physiol Genomics 50: 77-97, 2018.

19. Zhang CG, Jia ZQ, Li BH, Zhang H, Liu YN, Chen P, Ma KT and Zhou CY: beta-Catenin/TCF/LEF1 can directly regulate phenylephrine-induced cell hypertrophy and Anf transcription in cardiomyocytes. Biochem Biophys Res Commun 390: 258-262, 2009.

20. Liu Y,Zhou A, Zhao S, Huber WE and Li Q: Quadruple atrioventricular nodal pathways: involved in orthodromic atrioventricular reentrant tachycardia. Tex Heart Inst J 37: 706-709, 2010.

21. Huang L, He H, Liu Z, Liu D, Yin D and He M: Protective effects of isorhamnetin on cardiomyocytes against anoxia/ reoxygenation-induced injury is mediated by SIRT1.J Cardiovasc Pharmacol 67: 526-537, 2016.

22. Yu L, Li S, Tang X, Li Z, Zhang J, Xue X, Han J, Liu Y, Zhang Y, Zhang Y, et al: Diallyl trisulfide ameliorates myocardial ischemia-reperfusion injury by reducing oxidative stress and endoplasmic reticulum stress-mediated apoptosis in type 1 diabetic rats: Role of SIRT1 activation. Apoptosis 22: 942-954, 2017.

23. Chen X, Yan L, Guo Z, Chen Z, Chen Y, Li M, Huang C, Zhang X and Chen L: Adipose-derived mesenchymal stem cells promote the survival of fat grafts via crosstalk between the Nrf2 and TLR4 pathways. Cell Death Dis 7: e2369, 2016.

24. Deng S, Essandoh K, Wang X, Li Y, Huang W, Chen J, Peng J, Jiang DS, Mu X, Wang C, et al: Tsg101 positively regulates P62-Keap1-Nrf2 pathway to protect hearts against oxidative damage. Redox Biol 32: 101453, 2020.

25. Zhou H, Li N, Yuan Y, Jin YG, Guo H, Deng W and Tang QZ: Activating transcription factor 3 in cardiovascular diseases: A potential therapeutic target. Basic Res Cardiol 113: 37, 2018.

26. Ameri $\mathrm{K}$ and Harris AL: Activating transcription factor 4. Int J Biochem Cell Biol 40: 14-21, 2008.

27. Grumolato L, Liu G, Haremaki T, Mungamuri SK, Mong P, Akiri G, Lopez-Bergami P, Arita A, Anouar Y, Mlodzik M, et al: $\beta$-Catenin-independent activation of TCF1/LEF1 in human hematopoietic tumor cells through interaction with ATF2 transcription factors. PLoS Genet 9: e1003603, 2013.

This work is licensed under a Creative Commons Attribution-NonCommercial-NoDerivatives 4.0 International (CC BY-NC-ND 4.0) License. 